\title{
CAMBIOS CONCEPTUALES Y SIMBÓLICOS DE LAS SOCIEDADES RURALES: LA CARTA ETNOGRÁFICA COMO ELEMENTO REGULADOR
}

\author{
Conceptual and symbolic changes in rural societies: the ethnographic letter as a \\ regulator
}

\author{
FCo. Javier SAN Vicente \\ Universidad de Salamanca \\ Email: javiersanvicente@usal.es
}

Fecha de recepción: 6-III-2011

Fecha de aceptación: 4-IV-2011

\begin{abstract}
RESUMEN: Los modos de vida de las sociedades rurales tradicionales se encuentran en un franco proceso de desaparición. Bajo esta premisa empezamos a trabajar y planteamos un proyecto de investigación sobre la arquitectura del paisaje rural, el conocimiento del medio y la problemática de su regulación administrativa. Este trabajo nos serviría para estudiar, conocer, documentar e investigar en profundidad, desde un punto de vista etnológico, una cultura rural representada por algunas poblaciones del Norte de la comarca de La Ramajería; región que se localiza en el Noroeste de la provincia de Salamanca. Proponemos en nuestra investigación un ejemplo de carta etnológica que sirva como referencia a la hora del análisis de la catalogación administrativa de estos bienes, sin que ello sea excluyente de otras posibilidades, pero queriendo en todo momento y como finalidad prioritaria, fomentar la discusión sobre los distintos métodos o posibilidades que se pueden desarrollar en este tema.
\end{abstract}

Palabras Clave: Patrimonio, etnología, administración, tutela, fuente oral.

ABSTRACT: The livelihoods of traditional rural societies are in an open process of disappearing. Under this premise we started to work and propose a research project on rural landscape architecture, environmental awareness and issues of administrative regulation. This work would serve us to study, learn, document and investigate in depth from an ethnological point of view, a rural culture represented by some populations in the north of the region of La Ramajería; region that is located in the northwest of the province of Salamanca. We propose in our research ethnological a sample letter to serve 
as a reference when analyzing the administrative documentation of these goods, without it being exclusive of other possibilities, but willing at all times and as a priority objective, to promote discussion of different methods or possibilities that may develop in this area.

Keywords: Heritage, ethnology, administration, supervision, oral source.

\section{Presentación}

Los modos de vida de las sociedades rurales tradicionales se encuentran en un franco proceso de desaparición. Bajo esta premisa empezamos a trabajar y planteamos un proyecto de investigación sobre la arquitectura del paisaje rural, el conocimiento del medio y la problemática de su regulación administrativa. Este trabajo nos serviría para estudiar, conocer, documentar e investigar en profundidad, desde un punto de vista etnográfico, una cultura rural representada por algunas poblaciones del Norte de la comarca de La Ramajería; región que se localiza en el Noroeste de la provincia de Salamanca.

Se nos ha dado la oportunidad de estudiar los cambios marcados desde los entes administrativos en materias de ordenación territorial y económica. Así mismo hemos querido analizar el cambio conceptual y simbólico que está acaeciendo en estas sociedades, ante la introducción de valores representativos de las sociedades urbanas. Este modelo tiende a la globalización y homogeneización de las mentalidades y de los sistemas económicos, valorando su competitividad dentro del capitalismo actual. Esto ha obligado al abandono de métodos poco productivos y rentables, económicamente hablando, como ha sido la producción agrícola y ganadera tradicional que se ha desarrollado históricamente en estas localidades.

El cambio y la evolución profunda y rápida que se está dando en este ámbito, nos hace ser conscientes de la gravedad de la situación y de la oportunidad que aún tenemos de documentar no solo contextos materiales, de los que encontramos paralelismos en épocas históricas pasadas, sino de registrar los conceptos simbólicos y sicológicos que imbuían a estas comunidades. Cuya mentalidad y conocimiento del medio se contraponen a nuestra concepción global y urbana actual.

Las transformaciones acaecidas en el contexto rural, no solo de nuestra región, sino a nivel estatal, han producido la dispersión de conceptos sociales y materiales, que no solo servían de hilos conductores a la hora de explicar los distintos marcos locales en los que se encontraban, sino que además, eran portadores de informaciones sobre rasgos y conceptos históricos y arqueológicos.

Por todo ello pensamos que es necesario por parte de la administración, establecer una legislación propia a la hora de la catalogación y normalización administrativa de los bienes etnológicos. Es necesaria una sistematización de los inventarios y de los métodos de catalogación para clarificar y recoger un patrimonio cercano y en general poco valorado. 
Proponemos en nuestra investigación un ejemplo de carta etnológica que sirva como referencia a la hora del análisis de la catalogación administrativa de estos bienes, sin que ello sea excluyente de otras posibilidades, pero queriendo en todo momento y como finalidad prioritaria, fomentar la discusión sobre los distintos métodos o posibilidades que se pueden desarrollar en este tema.

A la hora de proponernos la realización de este proyecto se planteó un cronograma de actuación en dos fases, bajo la tutela de la profesora titular $\mathrm{M}^{\mathrm{a}}$ Carmen Sevillano San José del Departamento de Prehistoria, $\mathrm{H}^{\mathrm{a}}$ Antigua y Arqueología de la Facultad de Geografía e Historia de la Universidad de Salamanca. La primera fase, dada la ambición de la investigación, contempló la exposición de las bases metodológicas y contextuales del proyecto y al mismo tiempo sirvió de acicate para el análisis parcial de la etnología local, con lo que se ayudaba a una comprensión del entorno a través de conceptos generales y locales. Se estudió las implicaciones socio culturales, económicas, históricas y antropológicas de esta comunidad del Norte de La Ramajería, tratando de conferir hasta donde fuera posible un carácter interdisciplinar a este trabajo. Esta primera investigación se recoge parcialmente en el Trabajo de Grado inédito que llevó por título Arquitectura tradicional del noroeste de Salamanca: los pueblos del norte de Ramajería (SAN VICENTE, 2007) y en una monografía aún no publicada y financiada a través de una beca de investigación otorgada por la Fundación Villalar: Trabajo etnográfico del noroeste de la provincia de Salamanca.

En la actualidad se está llevando a cabo la segunda parte del proyecto dentro de una tesis doctoral de este mismo departamento. De esta primera investigación es de la que hablaremos es las líneas siguientes.

\section{ANTECEDENTES}

El mundo rural, se rige por conceptos cotidianos que envolvían de significados cognitivos a los espacios y estructuras materiales, los cuales tan solo con su registro descriptivo son imposibles de comprender.

La cultura de los pueblos existe por igual en su patrimonio material y en su cultura oral, que están íntimamente relacionadas con la evolución pausada pero constante que envuelve al mundo rural. Es una cultura representada en la mayoría de las ocasiones mediante características y adjetivos nada formales, en contra posición de la cultura historicista. Es un bagaje que lleva formándose, creciendo, y perdurando durante siglos, pero que a partir de mediados del siglo XX comienza a deteriorarse.

Ante la ruptura de este modo de vida, sin ninguna clase de transición, se hace necesario entablar diálogos que permitan documentar todos estos conocimientos. Esta cultura popular ha sufrido y sigue sufriendo un tremendo desgaste, la emigración y la modernización en el campo han supuesto una pérdida cultural considerable; las fiestas relacionadas con el ciclo agrícola y 
ganadero se han dejado de celebrar (tan solo se siguen conservando las más emblemáticas), los topónimos ante las parcelaciones o los repartos de tierras se encuentran en desuso,...

Es verdad que la supervivencia de las tradiciones o del folklore popular no está reñida con la mecanización y la tecnología, ya que siempre se debe intentar mejorar las condiciones de vida de cualquier medio, pero al mismo tiempo debemos de entender que se debe realizar una labor y concienciación social del verdadero valor de la cultura popular.

Ante este panorama la etnología aparece como una solución científica a la hora de registrar y documentar todos estos cambios, dándonos la ocasión de crear proyectos de cierta envergadura donde la seriedad y la síntesis metodológica sean las protagonistas. Demostrando la capacidad de esta disciplina de llevar a cabo un riguroso trabajo científico que sirva para rescatar los bienes materiales e inmateriales aún existentes, siendo viable la creación de proyectos donde se establezcan acciones de índole social o divulgativa.

Nos encontramos con un contexto legislativo en donde las políticas públicas articuladas y desarrolladas en el área del patrimonio cultural, están siendo una base sólida para el desarrollo sostenible de Castilla y León y las demás comunidades autónomas a las que se ha otorgado las competencias en esta materia. La evolución socio-económica de la región, la cohesión social junto con su identidad corren parejos a la divulgación, conocimiento y valoración de sus bienes patrimoniales. Esta línea de actuación en la práctica, se lleva desarrollándose desde los años ochenta del siglo pasado, a través de políticas turísticas basadas en la riqueza cultural de la comunidad; cuyos efectos se han proyectado hasta el presente.

El patrimonio etnológico, está representado y reconocido tanto en la Ley del Patrimonio Español de $1985^{1}$, así como en las distintas legislaciones posteriores como en las previsiones de la Ley 12/2002. y del reglamento de 2007/37 de Castilla y León. Estas leyes sentaban las bases jurídico-legislativas para el desarrollo de acciones de tutela del patrimonio etnológico en Castilla y León y la obligación de las administraciones públicas de actuar sobre él. Tras veintiséis años las políticas, actuaciones, medidas legislativas de protección y hasta la misma legalidad son ciertamente desconocidas, más aún, existe una generalizada ignorancia sobre estos bienes de nuestro patrimonio cultural no sólo por parte de la sociedad en general, sino lamentablemente, también entre los gestores públicos y colectivos académicos.

1 Artículo 1, punto 2 "Integran el Patrimonio Histórico Español los inmuebles y objetos muebles de interés artístico, histórico, paleontológico, arqueológico, etnográfico, científico o técnico. También forman parte del mismo el patrimonio documental y bibliográfico, los yacimientos y zonas arqueológicas, así como los sitios naturales, jardines y parques que tengan valor artístico, bistórico o antropológico". 
Sigue existiendo, administrativamente hablando, un desconocimiento o una falta de regulación por parte de los entes públicos de los bienes etnológicos. A la hora de la ordenación y gestión, se le aplican criterios de selección y valoración comúnmente utilizados para otras modalidades patrimoniales, sobre todo aquellas que han sido y son dominantes; no existen catalogaciones generales de bienes etnológicos, o si las hay son parciales solo de unos bienes en concreto, o de una zona determinada. Es verdad que la legislación, de manera amplia, aporta unas mínimas herramientas para la identificación y documentación de estos bienes, pero no son aprovechadas de forma práctica, no se avanza de forma eficaz en la protección y valoración de estas construcciones. Los criterios de análisis y tutela quedan sujetos a la sensibilidad y subjetividad de las entidades administrativas y a los pertinentes técnicos e inspectores.

Bajo esta tesitura nos pareció de interés armar un proyecto que aglutinase las diversas problemáticas señaladas anteriormente, estableciendo una localización específica donde se pudiese demostrar la vialidad y seriedad del área en el que queríamos trabajar. $\mathrm{Al}$ mismo tiempo queríamos construir y sintetizar una herramienta que reuniese las condiciones administrativas y prácticas a la hora de sistematizar el patrimonio etnológico, con el fin de proponer un modelo de acción que regule la catalogación etnológica con fines administrativos y que en la práctica llegue a tener como mínimo la importancia de las actuales Cartas Arqueológicas. Éstas sirven de base a la hora de llevar a cabo distintos trámites burocráticos, identificando mínimamente los elementos que son susceptibles de tener en cuenta antes de ejecutarse cualquier proyecto. La mejor forma de llevar a cabo esta investigación creemos que será la creación de una Carta Etnológica que agrupará todas estas necesidades y objetivos.

A estas premisas, se unió según avanzamos una última, que se encontraba fuera de los objetivos puramente científicos pero que creemos igualmente relevante, la de divulgación. Al iniciar el trabajo fuimos observando cómo las acciones sociales e investigaciones científicas, en ocasiones, se veían dentro de nuestro marco social de actuación como una injerencia exterior en su mundo, así como en sus valores y modos de vida. En muchas ocasiones las administraciones públicas y académicas establecen eventos cívicos y culturales que reivindican su propia cultura y tradiciones. Mientras que ellos mismos han vivido una evolución cultural, simbólica y conceptual promovida por estas mismas instituciones, basada en el sistema capitalista, urbano y global que les ha obligado al abandono y al cambio inconsciente de sus modelos sociales y económicos. Esta perspectiva, nos hizo plantearnos la necesidad y viabilidad de investigaciones como la nuestra, que además del aporte científico, tienen que contribuir a establecer bases de consenso que acerquen a los distintos estamentos sociales relacionados con este patrimonio. 


\section{LOCALIZACIÓN}

La aplicación de esta clase de investigación debía de contar con una localización geográfica acorde a las necesidades del proyecto, por ello se realizó una pequeña investigación previa, que nos llevó a establecer dentro de la provincia de Salamanca una zona de estudio. Tras la consulta de diversa bibliografía encontramos una comarca poco conocida y en un franco proceso de pérdida de identidad, denominada Ramajería. Se trata de un territorio cuyos núcleos rurales que lo conforman se encuentran sumergidos en diversos procesos de despoblamiento, marginación económica y administrativa. Siendo aún más notoria la marginación bibliográfica y documental, relacionada con todos los aspectos posibles, incluyendo el etnográfico.

Ante la problemática de la inexistencia documental y la amplitud territorial de la comarca, se abordó la elección de una serie de localidades de la zona norte de este territorio, como base de acción en el proyecto que se quería construir.

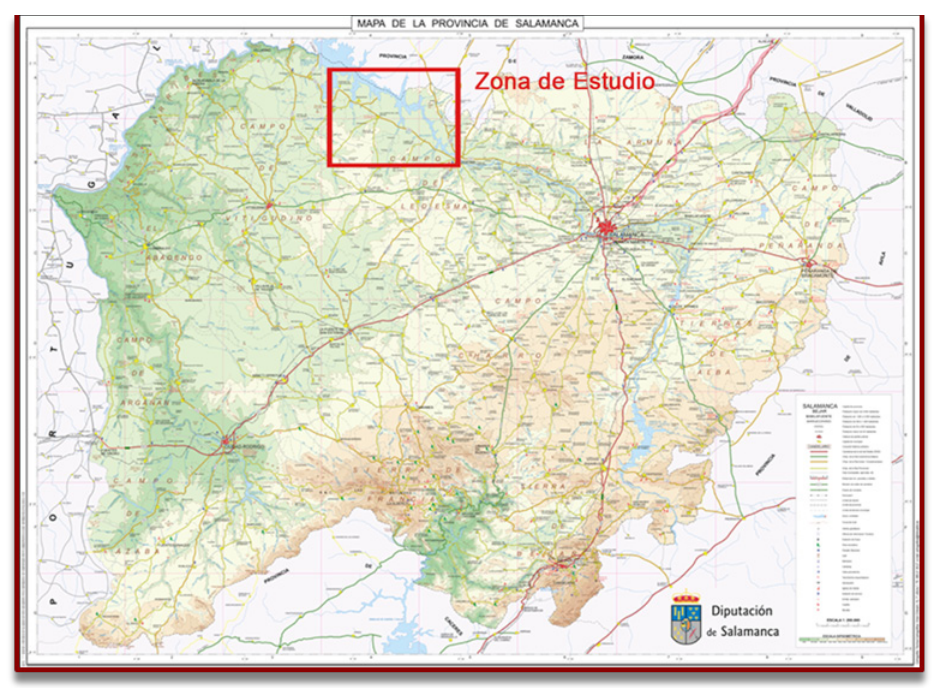

Mapa 1: Localización de la zona de estudio dentro de la Provincia de Salamanca 


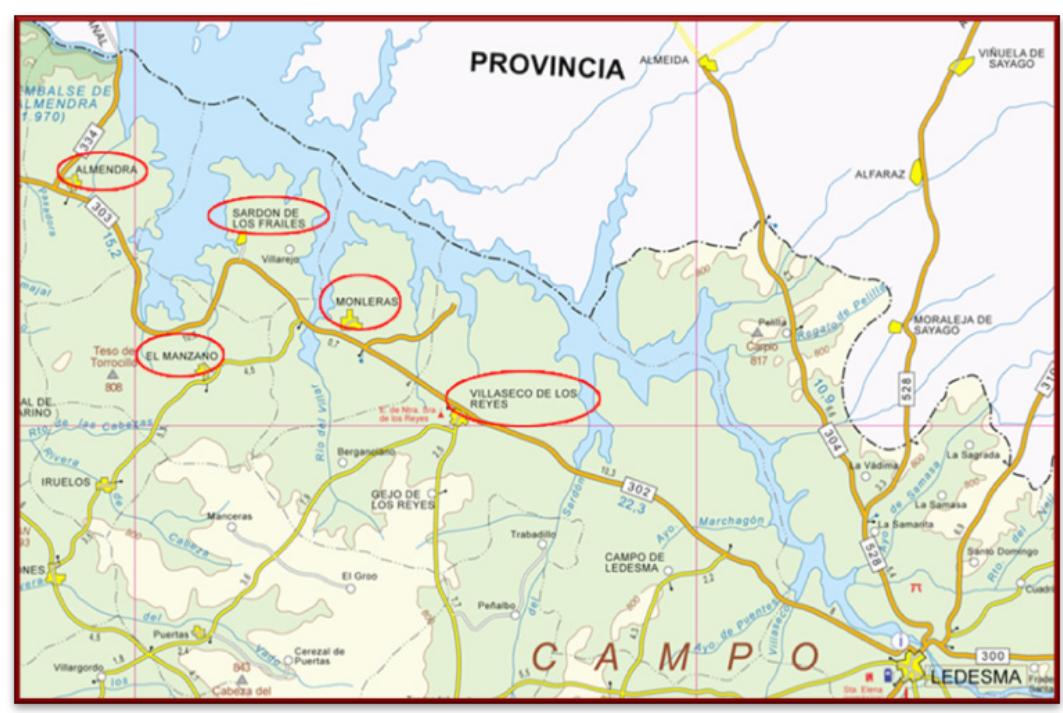

Mapa 2: Municipios en los que se desarrolló el trabajo.

\section{OBjetivos}

De forma general, conformamos tres líneas de actuación que resumieran los objetivos generales de nuestro proyecto: científica, administrativa y divulgativa. Además establecimos, a priori, una serie de objetivos específicos que avanzarían hacia la consecución final del proyecto que queríamos llevar a cabo:

1. La realización de un trabajo etnológico que permitiese demostrar la validez de esta ciencia a la hora de la comprensión de un territorio. Utilizaríamos el patrimonio etnológico como eje del mismo, pero sin olvidar los contextos físicos, económicos y sociales que le determinan.

2. Plantear la necesidad de una tutela y regulación por parte de la administración del patrimonio etnológico.

3. Conocer realmente qué se sabe, qué se recuerda y qué se opina acerca del patrimonio local, lo que implica al mismo tiempo concienciar a la población de la necesidad de un desarrollo cultural y natural en general, que a su vez lleve parejo un uso social. Mediante esta investigación se intentará involucrar al propio pueblo en el proyecto.

4. Descubrir la historia local, a través de la tradición de sus construcciones más emblemáticas y su contexto socio-económico.

5. Analizar y valorar todos los aspectos culturales y sociales de este mundo rural, relacionados con su patrimonio etnológico.

6. Estudiar e investigar la arquitectura rural menos conocida o secundaria, 
a través de las personas que la rodean partiendo de los conocimientos, la experiencia y el material que nos puedan aportar los vecinos de las localidades.

7. Establecer a través de las fuentes escritas y orales el perfil del patrimonio etnológico de este marco geográfico, intentando documentar sus fenómenos locales desde este punto de vista.

\section{Metodología}

A la hora de enfocar la solución práctica del proyecto que queríamos abordar, se estableció la realización de una metodología clara que nos llevará a la consecución de nuestro trabajo estableciendo una serie de pautas.

Los métodos que utilizamos en la presente investigación se basaron en una metodología básica de investigación etnológica que conformaron autores clásicos en esta materia. A ello se sumó la percepción metodológica de las distintas áreas en las que tuvimos que trabajar mientras desarrollábamos la investigación, cuya base obtuvimos a través de una bibliografía específica, como el tratamiento de las fuentes, la entrevista, la observación participante, fotografía... De la misma manera, la experiencia adquirida, nos ayudó a aprender y discernir la mejor manera y los mejores métodos a la hora de llevar a cabo los distintos aspectos del estudio. Dentro de este marco de actuación, hay que destacar que se utilizó la observación participante como base científica a la hora de la ejecución de este trabajo. La observación participante consiste en mantener un contacto continuo con la sociedad que se va a estudiar. Este contacto se resumió en la interacción con la gente de la zona durante un ciclo agrario completo, que comprendería algo más de un año. En donde se estuvo relación con todas las actividades diarias o puntuales que se desarrollan en la actualidad en este marco rural.

A la hora de abordar el proyecto y sus fases tuvimos muy en cuenta la concepción teórica antes expuesta, junto con los condicionantes que se le presuponen a cualquier trabajo o investigación, que en el caso de la etnografía se dan por supuestos, ya que es una ciencia que depende en gran manera del factor humano y sus variables. Esta disciplina es una ciencia donde el investigador debe ser flexible e improvisar en muchas ocasiones dentro del marco teórico establecido, pues sus fuentes están vivas y ello condiciona siempre en cierta manera la información que se obtiene (como ya hemos visto). De todas formas no hay que olvidar que hay igualmente una base escrita muy importante, de igual valor que la oral, que sirve junto con ésta para generar datos contrastados y fiables. Intentaremos aportar a través del siguiente esquema una idea general de la metodología empleada.

1. REFLEXIÓN, DOCUMENTACIÓN Y BIBLIOGRAFíA

$\mathrm{Al}$ tener ya establecido un contexto general de investigación, se realizó una búsqueda de bibliografía más específica relacionada con cada uno de los marcos generales que se iba a estudiar, como por ejemplo los contextos geográficos, históricos, o poblacionales, por citar algunas partes básicas de cualquier investigación. 


\subsection{Búsqueda y análisis planimétrico.}

Los dos formatos más comunes para trabajar en papel, son los planos a escala 1:25000 realizados por el Instituto Geográfico Nacional y la documentación catastral pertinente (plataforma catastral SigPac ${ }^{2}$ en internet), que nos aportó un conocimiento exhaustivo del terreno. Trabajamos a través de formatos digitales como .dwg, .cad, o .tiff en diferentes escalas.1:50000 y 1:25000. Utilizamos visores digitales como Google Earth, aunque sin olvidar la cartografía histórica.

\subsection{Vaciado toponímico.}

Se analizó toponímicamente los municipios y el territorio que se encuentra dentro del marco de la investigación, buscando cualquier término que hiciera referencia al patrimonio etnológico.

1.3 Preparación de fichas diagnóstico y de encuestas orales.

Se realizó una ficha diagnóstico que sirviera a la hora de la documentación de los bienes inmuebles. Se elaboraron encuestas orales, a modo de guión para facilitar la recogida de información.

\section{Cronograma de Trabajo.}

Se estableció un programa aproximado de las visitas físicas que se iban a realizar a los municipios.

\section{Trabajo de Campo.}

Planteado el cronograma se conformó un calendario de prospecciones visuales en función de la localización y proximidad de los objetivos.

Al trabajo de campo se acudió con las fichas y encuestas normalizadas, el sistema de localización, una grabadora para tomar cualquier información oral de la que se nos pudieran hacer partícipe y los equipos de video y foto. A la hora de abordar el trabajo de campo se tuvieron una serie de puntos claros:

- Se completó la ficha normalizada según el tema a abordar, de esa forma se pudieron tomar el máximo de datos con la mayor fiabilidad posible.

- Se fotografió y se realizaron pequeños videos para tener la máxima base documental visual a la hora de desarrollar el estudio.

- Se encontró y se entrevistó a informantes, a fin de conseguir información general y específica sobre el tema de estudio, por ejemplo si estamos trabajando sobre construcciones pudimos acercarnos a través de los entrevistados a la arquitectura de la zona, mediante la personificación de estas construcciones, ¿cómo se hicieron?, ¿quién las hacía?..., o cualquier información útil que en ese momento se nos

2 http://sigpac.mapa.es/fega/visor/ 
pueda aportar.

\section{Entrevistas y fuentes orales ${ }^{3}$}

Se realizaron entrevistas abiertas, y sistematizando en cualquier en todo momento las mismas y sus resultados.

\section{Observación participante (KENNETH,1990).}

Ésta método de documentación se hace necesario para poder entrever explicaciones emic ${ }^{4}$ de la sociedad que queremos intentar comprender.

\section{Memoria y conclusiones}

Una vez terminado la fase anterior se pasó a la elaboración de la memoria, a través del análisis exhaustivo de la bibliografía y del trabajo de campo. Se sistematizaron y digitalizaron los resultados de las informaciones vertidas en las fichas que se desarrollaron en el trabajo de campo, se analizó y se estableció el pertinente contexto teórico en el que se justificaban todos los aspectos metodológicos y prácticos vertidos en el desarrollo de la investigación. Se sistematizó y se sintetizó parte de la información traspasándolo a bases de datos ${ }^{5}$ digitales, mediante las cuales se pudo visualizar los resultados de una forma más coherente. Finalmente después del análisis de todos los datos, junto con la reflexión de los conceptos teóricos, se conformaron una serie de conclusiones que aglutinaron toda la experiencia adquirida a nivel práctico y teórico, que sirvieran de base para el desarrollo de la siguiente fase o investigación.

\section{REFLEXIONES FINALES}

\subsection{Cambios sociales y simbólicos}

Se ha conseguido llevar a cabo este trabajo, dando protagonismo a la memoria de las personas, que han sido con diferencia las mejores fuentes posibles. Hemos podido rescatar recuerdos, vivencias, anécdotas, datos, explicaciones socavadas en el tiempo, que han ayudado a recopilar, registrar, documentar, analizar y comprender el patrimonio etnológico local de estos pueblos. Se han recuperado términos, construcciones y concepciones inéditas de su concepto local, poniéndolas en relación con sus contextos generales.

Hemos comprobado como las construcciones tradicionales no son únicas de esta zona, pero sí lo son sus acepciones y significados. Ahí estriba la importancia de la investigación, que recoge además de la parte material,

3 Existen varias obras interesantes de este punto, que citamos en la bibliografía.

4 Etic, se refiere al mensaje emitido por cualquier observador de un marco social y cultural que no es el suyo a y emic, el mensaje literal que emite un observador que le ha sido aportado por un grupo social independiente al suyo. Ver Kenneth L. Pike 1990

5 Hay multitud de ejemplos y formatos digitales: Acces, Filemaker, Visual Basic, Oracle..... 
la parte conceptual y cognitiva, normalmente olvidada, que sirve para establecer paralelismos sociales con otros marcos rurales distintos.

Esta recopilación documental y oral nos posibilitó la realización de una valoración general del estado en el que se encuentra el patrimonio etnológico y un estudio de su contexto geográfico, histórico y social.

La situación real era y es clara, la mayoría de las construcciones se hallan en un estado de abandono más o menos acentuado y las demás se hallan en un proceso de transformación que nada tiene que ver con su evolución tradicional. En cuanto a los conceptos, acepciones y significados locales relacionados con este patrimonio, éstos han desaparecido; tan solo las personas de más edad son conscientes de los distintos conceptos cognitivos que contenían.

Son los elementos definitorios, los significados, los simbolismos y sus funciones; los que más riesgo corren en desaparecer y que esta sociedad rural acota a las distintas construcciones y actividades consideradas tradicionales. Toda esta riqueza se resume y se entrelaza en el patrimonio material e inmaterial.

El estado de abandono de costumbres, concepciones, construcciones, actividades, simbología..., no es lineal sino que existen variaciones dentro de la situación general. El concepto y la estructura de lo que podíamos denominar marco cultural tradicional ha desparecido, por lo menos tal y como venía evolucionando hasta hace menos de 50 años. La falta de planes de preservación y desarrollo cultural, urbano o paisajístico, que tengan en cuenta las connotaciones culturales, han hecho que se contrapongan elementos materiales e inmateriales tradicionales a las nuevas realidades y conceptos urbanos. Ha aparecido un sincretismo cultural donde la base actual es el modelo urbano, que se ha impuesto en el marco rural, transformando los característicos elementos materiales de este contexto, en simples elementos decorativos.

La aculturación de los modelos rurales hacia marcos urbanos desde el siglo pasado, es una de las conclusiones más rotundas que hemos podido comprobar en nuestra investigación. Encontramos, que los valores y conceptos sociales en la actualidad, son los mismos en estos municipios que en el ámbito urbano, pero al no existir ese marco aparece un desarraigo en el propio contexto local, un mundo que está a caballo entre dos universos uno que se acaba y otro que empieza.

Este contexto evolutivo acaba por establecer unos nuevos nexos de unión dentro de los núcleos rurales; las nuevas generaciones reniegan del marco simbólico preestablecido en sus hogares, dado que han ido creciendo en la sociedad de la información, mediante la que han tenido pleno acceso 
a los estereotipos y necesidades artificiales que la vida actual demanda, tan alejado de los sobrios cánones del medio rural.

Este panorama corroborado en el presente estudio, choca con el desarrollo y tratamiento cultural de este patrimonio en otras comunidades ${ }^{6}$ donde su evolución ha dado lugar a una revalorización de lo propio y de lo local, tanto a nivel social como científico.

Llega ser tan pobre la estima de las propias gentes de nuestra zona de estudio, a propósito de su propia cultura, que nos hemos encontrado casos donde las canciones y bailes que se enseñan en los talleres municipales han sustituido al propio cancionero o baile local porque "es más bonito" o "suena mejor".

En resumen el problema estriba en el cambio de mentalidad sufrido, por eso creemos que a la par de una preservación, documentación y estudio de estos valores tradicionales expuestos en este trabajo, existe una necesidad de realizar un gran trabajo de regulación, difusión y concienciación del valor de lo propio ante lo actual, pero no como valores irreconciliables, sino como una necesidad de entender nuestra cultura reciente para poder evolucionar y crecer como sociedad dentro de un marco hoy en día muy globalizado.

Es necesario inculcar esos valores sobre el terreno, a los entes municipales, las escuelas y el profesorado, así como a la sociedad rural tradicional. Si no se realiza, ocurren situaciones tan esperpénticas como ayuntamientos que reciben subvenciones relacionadas con el desarrollo cultural y por otro realizan escombreras a pocos metros de construcciones tradicionales. No es de recibo fomentar unos valores culturales determinados, sin que se extiendan a todos los elementos del patrimonio.

La aparición de algunas políticas locales basadas, en el desarrollo del patrimonio etnológico y cultural, por cuenta de algunos municipios, abre un camino que se ha de seguir hacia el convencimiento de que es necesario una tutela, gestión y divulgación en el mundo rural de su propia cultura, que en ella está uno de los mejores modelos posibles de supervivencia. No significa una vuelta atrás en cuanto a medios o comodidades pero si es necesario que vuelvan a valorar y recordar sus propios modelos económicos que han existido durante siglos, en ellos, como demuestran las acciones desarrolladas en otros territorios, tienen una base que les pueden llevar a establecer una identidad común forjada en el valor de lo propio.

\subsection{Las cartas y catalogaciones etnológicas.}

La elaboración de cartas etnográficas o catálogos de bienes inmuebles de carácter etnológico creemos que se puede enmarcar dentro de esta

\footnotetext{
6 Políticas culturales como la vasca, navarra, valenciana, balear o andaluza por citar las más relevantes, procuran en la práctica una política muy intensa sobre la protección y tutela del patrimonio etnológico.
} 
dinámica. Son las herramientas que estas entidades necesitan para la puesta en marcha de este tipo de acciones.

Desde hace años existe una política de protección preventiva del patrimonio que se viene realizando por el Servicio de Patrimonio de la Consejería de Cultura de la Junta de Castilla y León, a través de las normativas existentes ${ }^{7}$.

Transcurridos este tiempo en los que se ha ido elaborando diversos catálogos específicos sobre patrimonio etnológico: artesanal, pallozas, bodegas...(..), queremos proponer la realización de un catálogo etnológico de carácter general, que facilite el conocimiento a las administraciones locales y provinciales de las construcciones etnológicas existentes en los municipios de nuestra región. Estos inmuebles en la mayoría de las ocasiones no se encuentran recogidos dentro de las catalogaciones arqueológicas o históricas existentes. Así mismo por la variedad y la problemática de su regulación, se han reconocido tan solo lo más sobresalientes en contadas ocasiones, como Conjuntos Etnológicos. Existe una variedad muy numerosa de estos elementos susceptibles de ser inventariados y a los que la legislación actual protege y da cabida. El problema estriba en la falta de inventarios y catálogos de bienes etnológicos, que deben y pueden ser considerados con bienes susceptibles de ser inspeccionados ${ }^{8}$, tal y como en la legislación en materia de cultura consta.

En muchas ocasiones los pequeños municipios, demográfica y económicamente hablando, que se extienden por nuestra región, se encuentran con la incapacidad para conocer y ordenar el propio potencial cultural que se localiza dentro de sus términos municipales. Lo que conlleva el no ser conscientes del propio potencial económico y turístico, que ese patrimonio poco valorado y con el que han convivido toda su vida tiene hoy en día. Este tipo de catalogaciones les puede aportar una base de partida muy importante.

La elaboración de un catálogo o carta etnográfica pensamos que es el mejor instrumento de conservación indirecta o preventiva. Es un medio de conocimiento y valoración de los bienes culturales de un país, de una región o de una localidad cualquiera. La conservación de los bienes culturales comienza por su registro e identificación, tarea que se realiza por medio de los inventarios y catálogos. Tradicionalmente la inclusión de un bien cultural en un inventario supone su reconocimiento como objeto que exige tutela y protección. Los inventarios centran su acción en la identificación, descripción y ubicación del bien cultural, establecen una lista ordenada o sistemática de los mismos y son, por lo tanto, instrumentos de carácter más sumario o abreviado que los catálogos. Los catálogos, por el contrario, se diferencian

7 Decreto 37/2007 y previsiones del Decreto y previsiones de la Ley 12/2002.

8 Decreto 37/2007. Título VI . Artículo 138. 
de los inventarios en que centran su acción en la identificación, descripción y ubicación del bien cultural. Establecen una lista ordenada y sistemática de los mismos, e incluyen una valoración etnológica o cultural del inmueble, objeto o información oral en cuestión, por lo que son instrumentos que llevan asociada una profunda labor de investigación.

Además de las consideraciones administrativas que hemos expresado hasta el momento, la realización de este tipo de herramientas viene acompañada de un interés y una labor científica. La desaparición paulatina del marco rural tradicional refuerza la necesidad de ahondar en investigaciones de esta índole, que ayuden a regular, las nociones no solo materiales, sino simbólicas y conceptuales que envolvían a este patrimonio.

Estas construcciones formaban parte activa del conjunto social, económico y metafísico de cada municipio. A través de este tipo de proyectos se recupera, se investiga y se preserva una conciencia colectiva tradicional y característica de nuestra comunidad.

Queremos incluir dentro de nuestra catalogación no solo lo bienes inmuebles etnológicos más representativos de la región. Son susceptibles de ser inventariadas todas las construcciones que bajo nuestro criterio, alberguen unas connotaciones etnológicas claras y se enmarquen dentro de la arquitectura tradicional. Tenemos muy en cuenta que las características que conforman a los bienes etnológicos están en confrontación directa con los valores en los que se enmarca otro tipo de catalogaciones con carácter más historicista. Estas se supeditan a los valores tradicionales que suelen regir a la hora de documentar los bienes culturales, como son la singularización, escasez, antigüedad, permanencia, extrañeza, excepcionalidad, autenticidad, originalidad, valor estético, belleza, grandeza, monumentalidad(..).

En contrapartida los bienes etnológicos se pueden caracterizar por ser actuales, atemporales, plurales, diversos, cotidianos, modestos, vivos, evolutivos, inmateriales y materiales...de ahí las dificultades para su gestión, protección y conservación.

No podemos dejar de apuntar que esta propuesta no la ofrecemos como un proyecto independiente, sino que se engloba en una línea de actuación que se puede perfilar en diferentes direcciones, que lleve a la catalogación sistemática en otros municipios de este patrimonio ya sea inmueble, mueble o inmaterial.

\section{BiBLIOGRAFÍA}

BARANDIARÁN, J.M. “Guía para una encuesta etnográfica,. Cuadernos de Etnología y Etnografía de Navarra 20. Pamplona. 1975.

DÍAZ RADA, A. Etnografía y técnicas de investigación antropológica. Madrid, Universidad Nacional de Educación a Distancia, 2003. 
HAMMERSLEY, M. y ATKINSON, P. Etnografía. Métodos de investigación. Barcelona, Ediciones Paídos, 1994.

KENNETH, L. PIKE, y HARRIS, M. Emics and Etics:The Insider/ Outsider Debat, HeadlandN.Thomased., Londres, Sage Publications, 1990.

HAMMERD y WILDAUSKY, A "La entrevista semi-estructurada de final abierto. Aproximación a una guia operativa", Barcelona, Universidad de Barcelona, 1997, Historia, Antropología y Fuentes Orales, págs. 23-57.

KING DUNAWAYD, D. "La grabación de campo en la Historia Oral". Historia, Antropología y Fuentes Orales. Barcelona, Universidad de Barcelona, 1997, págs. 63-77.

MALINOSWKI, B. Los argonautas del Pacifico Occidental. Barcelona, Península, 2001.

ORTÍ, A. "La apertura y el enfoque cualitativo o estructural: la entrevista abierta semidirecta y la discusión de grupo. El análisis de la realidad social, Métodos y técnicas de investigación social. Madrid, Alianza, 1986.

SAN VICENTE VICENTE, Fco. J. "Guía básica para el conocimiento de la investigación etnográfica" [en línea]. Canto de la Musa. HYPERLINK "http://www.elcantodelamusa.com/docs/2010/diciembre/ doc4_guiabasica.pdf" http:// mmw.elcantodelamusa.com/docs/2010/diciembre/ doct guiabasica.pdf

SAN VICENTE VICENTE.Fco.J Arquitectura tradicional del noroeste de Salamanca: los pueblos del norte de la Ramajería. Trabajo de Grado Inédito. Universidad de Salamanca, 2007.

SÁNCHEZ GONZÁLEZ, J.M. "Los Ramajeros" III Encuentros de Etnología y Universidad. Universidad de Salamanca, 1988.

VV.AA. Patrimonio y Paisaje, Madrid, Abada, 2010. 
